\title{
Associations Between Maternal Hormonal Biomarkers and Maternal Mental and Physical Health of Very Low Birth Weight Infants
}

Asian/Pacific Island Nursing Journal

Volume 1(4): 149-161

(C)Author(s) 2016

http://digitalscholarship.unlv.edu/apin/

\author{
June Cho $^{\text {a }}$, Xiaogang Su ${ }^{\mathrm{b}}$, Vivien Phyllips ${ }^{\mathrm{c}}$, and Diane Holditch-Davis ${ }^{\mathrm{a}}$
}

\begin{abstract}
The purpose of this study was to determine whether maternal mental and physical health is associated with maternal testosterone and cortisol levels, parenting of very low birth weight infants, physical exercise, and White vs non-White race. A total of 40 mothers of very low birth weight infants were recruited from a neonatal intensive care unit at a University Hospital in the Southeast United States. Data were collected through a review of medical records, standardized questionnaires, and biochemical measurement. Maternal mental and physical health status using questionnaires as well as maternal testosterone and cortisol levels using an enzyme immunoassay were measured four times (birth, 40 weeks postmenstrual age [PMA], and 6 and 12 months [age of infant, corrected age]). General linear models showed that higher testosterone levels were associated with greater depressive symptoms, stress, and poorer physical health at 40 weeks PMA, and at 6 and 12 months. High cortisol levels were associated with greater anxiety at 40 weeks PMA; however, with better mental and physical health at 40 weeks PMA, and 6 and 12 months. Physical activity was associated with lower maternal perceived stress at 12 months. Maternal health did not differ by race, except anxiety, which was higher in White than non-White mothers after birth. As very low birth weight infants grew up, maternal physical health improved but mental health deteriorated. Testosterone and cortisol levels were found to be positively correlated in women but testosterone was more predictive of maternal mental and physical health than cortisol. Indeed testosterone consistently showed its associations with maternal health. Maternal stress might be improved through regular physical exercise.
\end{abstract}

Keywords: salivary testosterone and cortisol, maternal mental and physical health

Maternal mental and physical health are important not only for maternal well-being but also for optimal infant health and development. Mothers usually experience more difficulties when their infants are at risk for health and development problems such as very low birth weight (VLBW, birth weight $<1,500 \mathrm{~g}$ ) preterm infants because these infants require intensive maternal involvement to survive (Holditch-Davis, Schwartz, Black, \& Scher, 2007; Howe, Sheu, Wang, $\&$ Hsu, 2014). Mothers of VLBW preterm infants have an extra burden for parenting compared to mothers of normal birthweight full-term infants; thus they usually reported more problematic mental and physical health than other mothers (Cho, Holditch-Davis, \& Miles, 2008; Howe et al., 2014). Furthermore, such a burden is less often addressed among non-White mothers including Asian American mothers. This group of mothers is thus offered with proper treatment less often than Whites (Redshaw \& Henderson, 2016).

\footnotetext{
${ }^{a}$ Duke University, USA

${ }^{b}$ University of Texas at El Paso, USA

${ }^{c}$ University of Alabama at Birmingham
}

Corresponding Author:

June Cho, PhD, RN

Duke University

Email: june.cho@duke.edu 
Mental health has been assessed most often using self-report questionnaires. Use of hormonal biomarkers along with self-report could provide a more objective report than those with self-report alone. Among biomarkers, cortisol has been widely used to assess mental health status including stress and depressive symptoms because cortisol is considered a stress hormone. The end products of hypothalamus-pituitary-adrenal and hypothalamus-pituitary-gonadal axes, cortisol and testosterone, are partially released from adrenal glands. Because the levels of cortisol and testosterone are positively correlated in women (Cho, Carlo, Su, \& McCormick, 2012; Sarkar, Bergman, Fisk, O'Connor, \& Glover, 2007), we decided to measure both hormonal biomarkers to assess maternal mental health during early parenting of VLBW infants. The associations of cortisol and/or testosterone levels with maternal health problems should show more informative results than those without them.

High cortisol levels are also known to be negatively associated with women's physical health including hypertensive disorders, breast cancer, inflammatory disorders, and osteoporosis (Altindag et al., 2007; Hamer \& Steptoe, 2012; McGregor \& Antoni, 2009). Maternal cortisol levels usually increase during pregnancy and are associated with maternal and infant health problems such as short gestation and low birthweight (Bolten et al., 2011; Owen, Wood, Tomenson, Creed, \& Neilson, 2016). Similarly, high testosterone levels are known be related to physical health problems including ovarian cancer, diabetes, and rheumatoid arthritis (Chen et al., 2011; Cutolo, 2009; Soriguer et al., 2012). Elevated testosterone levels during pregnancy are associated with preeclampsia and intrauterine growth retardation (Carlsen, Jacobsen, \& Romundstad, 2006; Manikkam et al., 2004).

Poor maternal health appears to cause poor infant health (Brockington, Butterworth, \& Glangeaud-Freudenthal, 2016). Among many interventions, physical exercise has been found to be effective in reducing risks of anxiety, phobias, panic attacks, mood and stress disorders, and depressive symptoms (Janisse, Nedd, Escamilla, \& Nies, 2004; Penedo \& Dahn, 2005; Richardson et al., 2005) as well as in reducing the risks for diabetes, cardiovascular disease, obesity, and cancer (Brown, Burton, \& Rowan, 2007). Beneficial effects of physical exercise for mental health appear to be less promising than for physical health as cohort studies showed mixed relationships between physical exercise and depression (Bauman, 2004). Some studies showed a decreased risk of depression in individuals with regular physical exercise; whereas other studies showed no associations. In addition, which type of mental problems would benefit from regular physical exercise is unclear.

We compared maternal mental health between White and non-White mothers (including Asian Americans) and examined how maternal mental and physical health changed as their VLBW preterm infants grew up. We assumed that maternal mental, as well as physical health, would be improved as mothers become more familiar with child care and their daily lives (Penedo \& Dahn, 2005).

The purpose of this study was to examine whether (a) maternal testosterone and cortisol levels were associated with maternal mental and physical health, (b) maternal mental and physical health improved as their VLBW infants grew older, (c) physical exercise improved maternal mental and physical health, and ( $\mathrm{d}$ maternal mental and physical health differed between White and non-White mothers. The present study hypothesizes that maternal testosterone and cortisol levels would be predictive of maternal health, physical exercise would improve maternal health, maternal health would be improved when their VLBW infants grew up, and White mothers would have fewer mental and physical health problems than non-White mothers.

\section{Method}

We used a descriptive and comparative research design for this exploratory study. Data were collected through a review of medical records, standardized questionnaires, and biochemical measurement.

\section{Participants}

A convenience sample of 40 mothers of VLBW infants was recruited from the neonatal intensive care unit (NICU) of a tertiary medical center in the Southeastern United States. A post hoc power analysis revealed that a sample size of $N=40$ would allow us to detect an effect size as small as .2069 (Cohen's $f^{2}=R^{2} / 1-R^{2}$ ) with a power of $80 \%$ at the significance level $\alpha=.05$ using the multiple linear regression model with testosterone and cortisol included. The mother was recruited if she (a) had a birth within seven days to avoid infants who were unlikely to survive, (b) was older than 15 years to avoid mothers too young to understand the consent, (c) spoke English, and (d) were the primary caregiver of the infant. To reduce potential confounding effects, mothers were excluded if the (a) were dependent on narcotics or other drugs, (b) were HIV positive, or (c) had a documented serious medical or psychological problem such as cancer or postpartum psychosis. 


\section{Measures}

We obtained data through a review of medical records, standardized questionnaires, and biochemical measurement. Maternal data included demographic information, pregnancy history and birth record, and mental and physical health.

\section{Maternal Mental and Physical Health}

Maternal mental health was assessed at 2-4 time points. Data for depressive symptoms, anxiety, and perceived stress were collected at four time points (birth, 40 weeks postmenstrual age [PMA], 6 months, and 12 months of infant corrected age [CA]), the mental health survey was collected at three time points (40 weeks PMA, 6 months, and 12 months) as mothers had to be home after birth, and parenting stress was collected at two time points ( 6 and 12 months) as mother had to have parenting experiences with the infant at home. Maternal physical health was assessed at three time points (40 weeks PMA, 6 months, and 12 months CA) as mothers had to be home.

Twenty items proposed by the Center for Epidemiologic Studies Depression (CES-D; Radloff, 1977) were used to assess maternal depressive symptoms during the first year after birth. Scores range from 0-60, with higher scores indicating more depressive symptoms. Internal consistency using Cronbach's Alpha was reported as .85-.90 (Radloff, 1977). The internal consistency of the CES-D in this study ranged from .86-.90 across the four time points.

The six items under the State Anxiety subscale of the Spielberg State-Trait Anxiety Inventory (STAI; Marteau \& Bekker, 1992) were used to assess maternal anxiety. Scores range from 0-6, with higher scores indicating more anxiety. Internal consistency using Cronbach's Alpha was reported as .83 (van der Bij, de Weerd, Cikot, Steegers, \& Braspenning, 2003). The internal consistency of the STAI in this study ranged from .79-.90 across the four time points.

The 10-item version of the Cohen's Perceived Stress Scale (PSS-10; Andreou et al., 2011) was used to assess maternal perceived stress. Scores range from $0-10$, with higher scores indicating more perceived stress. Internal consistency using Cronbach's Alpha was reported as .73-.84 (Andreou et al., 2011; Lesage, Berjot, \& Deschamps, 2012). The internal consistency of the PSS in this study ranged from .82 to .87 across the four time points.

The 36-item Parenting Stress Index-Short Form (PSI/SF; (Haskett, Ahern, Ward, \& Allaire, 2006) was used to measure stress within the parentinfant relationship. Scores range from 36-180 and total score higher than 90 is considered an indication of risk for the well-being of the parent and the infant. Internal consistency using Cronbach's Alpha was reported as .78-.91 (Haskett et al., 2006).

The SF-12 Health Survey (SF-12; Ware, Kosinski, \& Keller, 1996) was used to identify maternal mental and physical health problems. The
SF-12 has been widely used and consists of mental and physical health composite scores that range from 0 100 , with a higher score indicating better health. Internal consistency using Cronbach's Alpha was reported as .76-.89 (King, Horowitz, Kassam, Yonas, \& Roberts, 2005; Ware et al., 1996). The internal consistency of the SF-12 in this study ranged from .56.58 across three time points.

The amount of weekly physical exercise was measure based on the frequency (how many days per week) and the level (strenuous, moderate, or mild) of physical exercise that ranges from 5-52, with a higher score indicating more physical exercise (Kim, Popkin, Siega-Riz, Haines, \& Arab, 2004).

\section{Saliva Sample Collection and Analysis}

Maternal testosterone and cortisol levels were measured using an enzyme immunoassay (EIA) at the University laboratory by a lab technician who was blinded to the maternal demographic information. All samples were measured twice for quality control at each time point (birth, 40 weeks PMA, 6 months, and 12 months CA).

\section{Testosterone}

We measured maternal testosterone levels in saliva because saliva contains free steroids that are unbound to proteins and more biochemically relevant because of easy accessibility to tissues (Rosner, Auchus, Azziz, Sluss, \& Raff, 2007). Maternal salivary testosterone levels were determined by EIA following the manufacturer's instructions (Salimetrics, LLC, State College, PA. https://www.salimetrics.com/assets/documents/1-

2402n.pdf). The intra- and inter-assay coefficients of variation, which express the precision and repeatability of the assay, were $2.5 \%$ and $5.6 \%$, respectively. Testosterone levels are higher in the morning than in the evening (Granger et al., 2003; Papacosta, Gleeson, \& Nassis, 2013), thus, we collected three samples from each mother between 9 AM to 12 PM to reduce diurnal rhythm of testosterone and to avoid extremely low testosterone levels in women. Maternal saliva samples at birth and 40 weeks PMA were collected at the NICU, whereas the samples at 6 and 12 months CA were collected either at the Newborn Follow-Up clinic or at the Research Project Office.

\section{Cortisol}

Cortisol levels vary diurnally and are higher in the morning than in the evening (Mezzullo et al., 2016) so we used the saliva samples collected for testosterone measurement. Salivary cortisol levels were determined by EIA following the manufacturer's instructions (Salimetrics, LLC, State College, PA. https://www.salimetrics.com/assets/ documents/13002 n.pdf). The intra- and inter-assay coefficients of variation were $3.3 \%$ and $3.7 \%$, respectively. 


\section{Procedure}

This study was approved by the Institutional Review Board at the university. On a daily basis, a research nurse identified eligible mothers for the study through the NICU admission log. The research nurse discussed the study with the mothers, and the informed consent form was given to the mothers for their review. After maternal consent, a research nurse reviewed the medical records of the mothers and interviewed mothers to complete the data forms at the NICU. The research nurse collected maternal saliva samples and asked mothers to complete the CES-D, STAI, and PSS after birth and at 40 weeks PMA. At 40 weeks PMA, the research nurse and the research assistant updated maternal demographic and contact information for the follow-up visit.

Saliva from mothers was obtained by having them use a straw to expectorate into a small tube one hour before or after any oral intake. Three samples of saliva $(0.1 \mathrm{ml}$ each with a 15 -min interval) from each mother were collected within a two-hour period to minimize a pulsatile and diurnal secretion pattern of hormonal markers (Rosner et al., 2007). We collected saliva samples without using collection devices such as mouth swab or cotton roll because the devices may introduce assay artifacts. All saliva samples were placed on ice to transfer to the laboratory at the university and stored in a $-80{ }^{\circ} \mathrm{C}$ freezer until assayed.

\section{Follow-Up Data Collection at 6 and 12 Months CA}

Mothers were asked to visit either the Newborn Follow-Up clinic or the Research Project Office when the infants were 6 and 12 months CA. During the 6- and 12- month visits, the research nurse and the research assistant collected saliva samples and updated the demographic information of mother. The research nurse asked mothers to complete the CES-D, STAI, PSS, 12-item Short Form Health Survey Mental Composite Score (SFMCS), and PSI to assess maternal mental health status as well as to complete the 12item Short Form Health Survey Physical Composite Score (SFPCS) and physical exercise to assess maternal physical health status at 6 and 12 months.

Table 1. Maternal Characteristics and Steroid Hormonal Levels

\begin{tabular}{|c|c|c|c|c|c|}
\hline Variable & $n$ & Minimum & Maximum & $M$ & $S D$ \\
\hline Age & 40 & 18 & 38 & 25.88 & 5.765 \\
\hline Gravida & 40 & 1 & 6 & 2.53 & 1.396 \\
\hline Parity & 40 & 1 & 5 & 1.88 & 1.067 \\
\hline Race: White (\%) & 40 & & & 60 & \\
\hline Married (\%) & 40 & & & 45 & \\
\hline Education: < High school $(\%)$ & 40 & & & 45 & \\
\hline Insurance: Public (\%) & 40 & & & 60 & \\
\hline Body mass index & 40 & 20.88 & 49.43 & 34.26 & 6.930 \\
\hline Pregnancy complications & 40 & 0 & 3 & 1.08 & .694 \\
\hline Labor complications & 40 & 2 & 5 & 4.43 & .813 \\
\hline Prenatal steroid $(\%)$ & 40 & & & 95 & \\
\hline Delivery: Vaginal (\%) & 40 & & & 30 & \\
\hline Testosterone at birth & 40 & 12.65 & 158.56 & 64.27 & 34.328 \\
\hline Cortisol at birth & 37 & .03 & .57 & .13 & .147 \\
\hline Testosterone at 40 weeks PMA & 39 & 24.99 & 110.09 & 52.71 & 18.869 \\
\hline Cortisol at 40 weeks PMA & 39 & .036 & .58 & .23 & .124 \\
\hline Testosterone at 6 months & 34 & 26.99 & 124.62 & 62.56 & 23.212 \\
\hline Cortisol at 6 months & 34 & .07 & 1.47 & .29 & .251 \\
\hline Testosterone at 12 months & 34 & 23.43 & 128.63 & 55.50 & 22.020 \\
\hline Cortisol at 12 months & 34 & .03 & .35 & .17 & .080 \\
\hline
\end{tabular}

Note. Pregnancy complications = number of medical complications during pregnancy, Labor complications $=$ number of medical complications at labor/birth, PMA = postmenstrual age

\section{Data Analysis}

General linear model was the main analytic tool used for determining whether maternal salivary testosterone and cortisol levels were associated with maternal mental and physical health as well as maternal mental health were associated with physical exercise. Pearson correlation coefficients were used to determine the relationships among each maternal mental and physical health at the four time points. We used $t$-tests to compare maternal mental and physical health status between non-White and White mothers. Multiple comparisons were made in testing our hypotheses; however, we did not make any multiplicity adjustment owing to the exploratory nature of this study. This is because $p$-value adjustments are calculated based on how many tests are to be considered, and that number has been defined arbitrarily and variably especially in exploratory studies, as pointed out by (Feise, 2002) among others. 
Table 2. Associations Between Maternal Hormonal Markers (Testosterone and Cortisol Levels) and Maternal Mental and Physical Health During the First Year After Birth

\begin{tabular}{|c|c|c|c|c|c|}
\hline DV & Parameter & $\beta$ & $S E$ & $t$ & $p$ \\
\hline \multirow[t]{3}{*}{ CESD at 40 weeks PMA } & Intercept & 8.015 & 2.892 & 2.771 & .011 \\
\hline & Testosterone at birth & .085 & .035 & 2.454 & .022 \\
\hline & Cortisol at birth & -10.339 & 7.843 & -1.318 & .200 \\
\hline \multirow[t]{3}{*}{ PSS at 40 weeks PMA } & Intercept & 11.303 & 2.902 & 3.895 & .001 \\
\hline & Testosterone at birth & .078 & .035 & 2.225 & .036 \\
\hline & Cortisol at birth & -9.412 & 7.869 & -1.196 & .243 \\
\hline \multirow[t]{3}{*}{ SFPCS at 40 weeks PMA } & Intercept & 55.761 & 2.407 & 23.162 & .000 \\
\hline & Testosterone at birth & -.105 & .029 & -3.629 & .001 \\
\hline & Cortisol at birth & -11.560 & 6.528 & -1.771 & .089 \\
\hline \multirow[t]{3}{*}{ SFMCS at 40 weeks PMA } & Intercept & 51.201 & 3.893 & 13.151 & .000 \\
\hline & Testosterone at birth & -.095 & .047 & -2.032 & .053 \\
\hline & Cortisol at birth & 22.881 & 10.558 & 2.167 & .040 \\
\hline \multirow[t]{3}{*}{ Physical Exercise at 12 months } & Intercept & 9.733 & 3.985 & 2.443 & .022 \\
\hline & Testosterone at birth & -.052 & .048 & -1.084 & .289 \\
\hline & Cortisol at birth & 26.815 & 10.805 & 2.482 & .020 \\
\hline \multirow[t]{3}{*}{ PSS at 12 months } & Intercept & 4.379 & 3.953 & 1.108 & .278 \\
\hline & Testosterone at birth & .200 & .071 & 2.840 & .008 \\
\hline & Cortisol at birth & 3.186 & 10.501 & .303 & .764 \\
\hline \multirow{3}{*}{ STAI at 40 weeks PMA } & Intercept & 3.063 & 1.729 & 1.771 & .088 \\
\hline & Testosterone at 40 weeks PMA & .047 & .031 & 1.520 & .140 \\
\hline & Cortisol at 40 weeks PMA & 15.696 & 4.593 & 3.417 & .002 \\
\hline \multirow[t]{3}{*}{ STAI at 6 months } & Intercept & 4.098 & 2.173 & 1.886 & .070 \\
\hline & Testosterone at 40 weeks PMA & .051 & .039 & 1.305 & .203 \\
\hline & Cortisol at 40 weeks PMA & 12.262 & 5.774 & 2.124 & .043 \\
\hline \multirow[t]{3}{*}{ SFPCS at 6 months } & Intercept & 42.810 & 5.434 & 7.878 & .000 \\
\hline & Testosterone at 40 weeks PMA & -.062 & .097 & -.637 & .530 \\
\hline & Cortisol at 40 weeks PMA & 31.940 & 14.436 & 2.212 & .036 \\
\hline \multirow[t]{3}{*}{ SFMCS at 6 months } & Intercept & 64.024 & 5.545 & 11.547 & .000 \\
\hline & Testosterone at 40 weeks PMA & -.227 & .099 & -2.289 & .030 \\
\hline & Cortisol at 40 weeks PMA & -19.366 & 14.730 & -1.315 & .200 \\
\hline \multirow[t]{3}{*}{ PSI at 12 months } & Intercept & 39.680 & 9.959 & 3.984 & .000 \\
\hline & Testosterone at 40 weeks PMA & .400 & .178 & 2.253 & .033 \\
\hline & Cortisol at 40 weeks PMA & 8.919 & 26.455 & .337 & .739 \\
\hline \multirow[t]{3}{*}{ Physical Exercise at 6 months } & Intercept & 4.627 & 5.069 & .913 & .369 \\
\hline & Testosterone at 40 weeks PMA & .059 & .077 & .764 & .452 \\
\hline & Cortisol at 40 weeks PMA & 24.965 & 6.790 & 3.677 & .001 \\
\hline \multirow[t]{3}{*}{ Physical Exercise at 12 months } & Intercept & -.075 & 5.784 & -.013 & .990 \\
\hline & Testosterone at 6 months & .093 & .088 & 1.055 & .301 \\
\hline & Cortisol at 6 months & 21.024 & 7.748 & 2.714 & .011 \\
\hline
\end{tabular}

Note. CES-D $=$ the Center for Epidemiologic Studies Depression, DV = dependent variable, PSS = Perceived Stress Scale, STAI $=$ State-Trait Anxiety Index, SFMCS = the 12 Item Short Form Health Survey Mental Composite Score, PSI = Parenting Stress Index, SFPCS = the 12 Item Short Form Health Survey Physical Composite Score, PMA = postmenstrual age

\section{Results}

\section{Maternal Demographic Characteristics and Steroid Hormonal Levels}

Of the 40 mothers, $45 \%$ had more than a high school education, $30 \%$ were married, $60 \%$ were nonwhite, $70 \%$ had a cesarean section, and $60 \%$ had public insurance. Mean maternal age was 26 years and $95 \%$ of mothers completed a prenatal glucocorticoid treatment (see Table 1). Means of gravida and parity were 2.5 and 1.9, respectively, and the mean of BMI was $34.3 \mathrm{~kg} / \mathrm{m}^{2}$ before delivery. Eighty-three percent of mothers had medical complications during pregnancy such as chorioamnionitis and antepartum hemorrhage, and $100 \%$ mothers had medical complications at birth such as premature prolonged rupture of membrane and antibiotics use because of premature prolonged rupture of membrane. The mean of maternal testosterone levels at the four time points 
were $64.3 \mathrm{pg} / \mathrm{ml}, 52.7 \mathrm{pg} / \mathrm{ml}, 62.6 \mathrm{pg} / \mathrm{ml}$, and 55.5 $\mathrm{pg} / \mathrm{ml}$, respectively, whereas the mean of maternal cortisol levels at the four time points were $0.13 \mu \mathrm{g} / \mathrm{dL}$, $0.23 \mu \mathrm{g} / \mathrm{dL}, 0.29 \mu \mathrm{g} / \mathrm{dL}$, and $0.17 \mu \mathrm{g} / \mathrm{dL}$, respectively. Maternal demographic characteristics and the overall means of testosterone and cortisol levels at the four time points did not differ between non-White mothers and White mothers. Prenatal glucocorticoid treatment did not affect maternal testosterone and cortisol levels.

\section{Associations of Maternal Steroid Hormonal Levels and Maternal Mental and Physical Health}

As shown in Table 2, high maternal testosterone levels were consistently associated with maternal mental health problems as well as physical health problems. High maternal testosterone levels at birth were associated with greater maternal depressive symptoms at 40 weeks PMA $(\beta=.085, S E=.035, p=$ $.03)$ and greater maternal perceived stress at 12 months $(\beta=.078, S E=.035, p=.04)$, whereas high maternal testosterone levels at birth were associated with worse physical health at 40 weeks PMA ( $\beta=-.105, S E=.029$, $p=.001)$. High maternal testosterone levels at 40 weeks PMA were associated with greater maternal perceived stress $(\beta=.200, S E=.071, p=.008)$ as well as parenting stress at 12 months $(\beta=.400, S E=.178, p$ $=.04$ ), whereas high maternal testosterone levels at 40 weeks PMA were associated with smaller maternal mental composite score on the SF-12, which indicated worse mental health, at 6 months $(\beta=-.227, S E=.099$, $p=.03)$.

Unlike testosterone levels, high maternal cortisol levels were not consistently associated with problematic maternal mental and physical health. High maternal cortisol levels at birth were associated with a higher maternal mental health composite score on the SF-12, which indicated better mental health, at 40 weeks PMA ( $\beta=22.881, S E=10.558, p=.04)$ as well as more physical exercise at 12 months $(\beta=26.815, S E$ $=10.805, p=.02$ ). High maternal cortisol levels at 40 weeks PMA were associated with greater anxiety at 40 weeks PMA ( $\beta=15.696, S E=4.593, p=.002)$ and 6 months $(\beta=12.262, S E=5,774, p=.05)$, and greater maternal physical composite score on the SF-12, which indicated better physical health, at 6 months ( $\beta$ $=31.940, S E=14.436, p=.04)$. High maternal cortisol levels at 6 months were associated with more physical exercise $(\beta=24.965, S E=6.790, p=.001)$. Neither maternal testosterone nor cortisol levels at 12 months were associated with any maternal mental and physical health problems at 12 months.

Table 3. Maternal Mental and Physical Health During the First Year After Birth

\begin{tabular}{|c|c|c|c|c|c|}
\hline Variable & $n$ & Minimum & Maximum & $M$ & $S D$ \\
\hline CESD at birth & 40 & 2 & 42 & 13.75 & 10.233 \\
\hline CESD at 40 weeks PMA & 38 & 2 & 43 & 13.00 & 9.693 \\
\hline CESD at 6 months & 32 & 1 & 34 & 12.13 & 9.557 \\
\hline CESD at 12 months & 34 & 2 & 43 & 11.94 & 9.850 \\
\hline PSS at birth & 40 & 0 & 29 & 14.43 & 7.555 \\
\hline PSS at 40 weeks PMA & 38 & 0 & 31 & 13.39 & 7.992 \\
\hline PSS at 6 months & 32 & 4 & 30 & 13.78 & 7.255 \\
\hline PSS at 12 months & 34 & 0 & 34 & 14.79 & 6.839 \\
\hline STAI at birth & 40 & 6 & 22 & 10.48 & 4.095 \\
\hline STAI at 40 weeks PMA & 38 & 6 & 22 & 9.29 & 4.113 \\
\hline STAI at 6 months & 32 & 6 & 21 & 9.31 & 3.711 \\
\hline STAI at 12 months & 34 & 6 & 17 & 9.68 & 3.400 \\
\hline SFMCS at 40 weeks PMA & 38 & 24.7 & 65.4 & 49.13 & 10.536 \\
\hline SFMCS at 6 months & 32 & 24.7 & 60.7 & 48.47 & 9.528 \\
\hline SFMCS at 12 months & 34 & 25.8 & 62.5 & 47.91 & 9.362 \\
\hline PSI at 6 months & 32 & 36 & 129 & 57.34 & 18.988 \\
\hline PSI at 12 months & 34 & 37 & 103 & 62.53 & 18.042 \\
\hline SFPCS at 40 weeks PMA & 38 & 25.1 & 57.3 & 46.60 & 6.9870 \\
\hline Physical Exercise at 40 weeks PMA & 38 & 5 & 48 & 16.97 & 13.351 \\
\hline SFPCS at 6 months & 32 & 26.1 & 65.9 & 46.82 & 9.003 \\
\hline Physical Exercise at 6 months & 31 & 5 & 40 & 15.48 & 11.483 \\
\hline SFPCS at 12 months & 34 & 28.3 & 62.7 & 48.48 & 8.028 \\
\hline Physical Exercise at 12 months & 34 & 5 & 46 & 11.71 & 11.790 \\
\hline
\end{tabular}

Note. CES-D = the Center for Epidemiologic Studies Depression, PSS = Perceived Stress Scale, STAI = State-Trait Anxiety Index, SFMCS $=$ the 12 Item Short Form Health Survey Mental Composite Score, PSI $=$ Parenting Stress Index, SFPCS $=$ the 12 Item Short Form Health Survey Physical Composite Score, PMA = postmenstrual age 
Table 4. Physical Exercise and Maternal Mental Health at 40 weeks PMA, and 6 and 12 Months

\begin{tabular}{|c|c|c|c|c|c|}
\hline DV & Parameter & $\beta$ & $S E$ & $t$ & $p$ \\
\hline \multirow{4}{*}{ CESD at 40 weeks PMA } & Intercept & 11.112 & 2.556 & 4.347 & .000 \\
\hline & Physical Exercise at 40 weeks & .114 & .118 & .963 & .345 \\
\hline & Physical Exercise at 6 months & .171 & .150 & 1.137 & .266 \\
\hline & Physical Exercise at 12 months & -.317 & .161 & -1.964 & .060 \\
\hline \multirow{4}{*}{ CESD at 6 months } & Intercept & 8.880 & 3.330 & 2.667 & .013 \\
\hline & Physical Exercise at 40 weeks & .240 & .154 & 1.559 & .131 \\
\hline & Physical Exercise at 6 months & .196 & .196 & 1.001 & .326 \\
\hline & Physical Exercise at 12 months & -.352 & .210 & -1.675 & .106 \\
\hline \multirow{4}{*}{ CESD at 12 months } & Intercept & 10.855 & 3.564 & 3.046 & .005 \\
\hline & Physical Exercise at 40 weeks & .112 & .165 & .678 & .504 \\
\hline & Physical Exercise at 6 months & .081 & .210 & .388 & .701 \\
\hline & Physical Exercise at 12 months & -.199 & .225 & -.887 & .383 \\
\hline \multirow[t]{4}{*}{ PSS at 40 weeks PMA } & Intercept & 17.173 & 2.650 & 6.481 & .000 \\
\hline & Physical Exercise at 40 weeks & .018 & .123 & .148 & .883 \\
\hline & Physical Exercise at 6 months & -.036 & .156 & -.233 & .817 \\
\hline & Physical Exercise at 12 months & -.272 & .167 & -1.628 & .116 \\
\hline \multirow[t]{4}{*}{ PSS at 6 months } & Intercept & 13.210 & 2.626 & 5.030 & .000 \\
\hline & Physical Exercise at 40 weeks & .124 & .121 & 1.019 & .318 \\
\hline & Physical Exercise at 6 months & .142 & .154 & .918 & .367 \\
\hline & Physical Exercise at 12 months & -.300 & .166 & -1.811 & .082 \\
\hline \multirow[t]{4}{*}{ PSS at 12 months } & Intercept & 14.533 & 2.427 & 5.987 & .000 \\
\hline & Physical Exercise at 40 weeks & .196 & .112 & 1.747 & .092 \\
\hline & Physical Exercise at 6 months & .088 & .143 & .613 & .545 \\
\hline & Physical Exercise at 12 months & -.336 & .153 & -2.193 & .037 \\
\hline \multirow[t]{4}{*}{ STAI at 40 weeks PMA } & Intercept & 8.215 & 1.242 & 6.616 & .000 \\
\hline & Physical Exercise at 40 weeks & .055 & .057 & .965 & .343 \\
\hline & Physical Exercise at 6 months & .081 & .073 & 1.105 & .279 \\
\hline & Physical Exercise at 12 months & -.128 & .078 & -1.641 & .113 \\
\hline \multirow[t]{4}{*}{ STAI at 6 months } & Intercept & 8.541 & 1.390 & 6.143 & .000 \\
\hline & Physical Exercise at 40 weeks & .037 & .064 & .570 & .574 \\
\hline & Physical Exercise at 6 months & .113 & .082 & 1.384 & .178 \\
\hline & Physical Exercise at 12 months & -.134 & .088 & -1.525 & .139 \\
\hline \multirow{4}{*}{ STAI at 12 months } & Intercept & 9.174 & 1.236 & 7.422 & .000 \\
\hline & Physical Exercise at 40 weeks & .035 & .057 & .617 & .542 \\
\hline & Physical Exercise at 6 months & .110 & .073 & 1.509 & .143 \\
\hline & Physical Exercise at 12 months & -.138 & .078 & -1.776 & .088 \\
\hline \multirow[t]{4}{*}{ SFMCS at 40 weeks PMA } & Intercept & 46.460 & 3.427 & 13.557 & .000 \\
\hline & Physical Exercise at 40 weeks & .000 & .158 & .001 & .999 \\
\hline & Physical Exercise at 6 months & -.179 & .202 & -.890 & .382 \\
\hline & Physical Exercise at 12 months & .381 & .216 & 1.762 & .090 \\
\hline \multirow[t]{4}{*}{ SFMCS at 6 months } & Intercept & 48.846 & 3.688 & 13.243 & .000 \\
\hline & Physical Exercise at 40 weeks & -.105 & .171 & -.613 & .545 \\
\hline & Physical Exercise at 6 months & -.103 & .217 & -.473 & .640 \\
\hline & Physical Exercise at 12 months & .242 & .233 & 1.042 & .307 \\
\hline \multirow[t]{4}{*}{ SFMCS at 12 months } & Intercept & 51.965 & 3.439 & 15.109 & .000 \\
\hline & Physical Exercise at 40 weeks & -.247 & .159 & -1.554 & .132 \\
\hline & Physical Exercise at 6 months & -.198 & .202 & -.981 & .336 \\
\hline & Physical Exercise at 12 months & .260 & .217 & 1.198 & .242 \\
\hline \multirow[t]{4}{*}{ PSI at 6 months } & Intercept & 65.441 & 7.313 & 8.949 & .000 \\
\hline & Physical Exercise at 40 weeks & -.239 & .338 & -.706 & .486 \\
\hline & Physical Exercise at 6 months & -.178 & .430 & -.415 & .681 \\
\hline & Physical Exercise at 12 months & -.100 & .461 & -.217 & .830 \\
\hline \multirow[t]{4}{*}{ PSI at 12 months } & Intercept & 59.866 & 6.166 & 9.709 & .000 \\
\hline & Physical Exercise at 40 weeks & .108 & .285 & .378 & .709 \\
\hline & Physical Exercise at 6 months & .472 & .363 & 1.303 & .204 \\
\hline & Physical Exercise at 12 months & -.616 & .389 & -1.584 & .125 \\
\hline
\end{tabular}

Note. CES-D = the Center for Epidemiologic Studies Depression, DV = dependent variable, PMA = postmenstrual age, PSI = Parenting Stress Index, PSS = Perceived Stress Scale, SFMCS = the 12 Item Short Form Health Survey Mental Composite Score, SFPCS $=$ the 12 Item Short Form Health Survey Physical Composite Score, STAI = State-Trait Anxiety Index 
Table 5. Maternal Mental and Physical Health Differences Between Non-White Mothers $(N-W)$ and White Mothers (W)

\begin{tabular}{|c|c|c|c|c|c|c|c|}
\hline Variable & Race & $n$ & $M$ & $S D$ & $t$ & $d f$ & $p$ \\
\hline \multirow[t]{2}{*}{ CESD at birth } & N-W & 24 & 12.67 & 9.635 & -.817 & 38 & .419 \\
\hline & $\mathrm{W}$ & 16 & 15.38 & 11.189 & & & \\
\hline \multirow[t]{2}{*}{ CESD at 40 weeks PMA } & $\mathrm{N}-\mathrm{W}$ & 23 & 11.87 & 6.771 & -.888 & 36 & .381 \\
\hline & $\mathrm{W}$ & 15 & 14.73 & 13.074 & & & \\
\hline \multirow[t]{2}{*}{ CESD at 6 months } & $\mathrm{N}-\mathrm{W}$ & 19 & 11.47 & 8.501 & -.460 & 30 & 649 \\
\hline & $\mathrm{W}$ & 13 & 13.08 & 11.221 & & & \\
\hline \multirow[t]{2}{*}{ CESD at 12 months } & $\mathrm{N}-\mathrm{W}$ & 21 & 12.48 & 9.688 & .397 & 32 & .694 \\
\hline & $\mathrm{W}$ & 13 & 11.08 & 10.444 & & & \\
\hline \multirow{2}{*}{ PSS at birth } & $\mathrm{N}-\mathrm{W}$ & 24 & 13.67 & 7.750 & -.774 & 38 & .444 \\
\hline & $\mathrm{W}$ & 16 & 15.56 & 7.348 & & & \\
\hline \multirow[t]{2}{*}{ PSS at 40 weeks PMA } & $\mathrm{N}-\mathrm{W}$ & 23 & 13.48 & 7.988 & .079 & 36 & .938 \\
\hline & $\mathrm{W}$ & 15 & 13.27 & 8.276 & & & \\
\hline \multirow[t]{2}{*}{ PSS at 6 months } & $\mathrm{N}-\mathrm{W}$ & 19 & 14.47 & 7.329 & .647 & 30 & .523 \\
\hline & $\mathrm{W}$ & 13 & 12.77 & 7.316 & & & \\
\hline \multirow[t]{2}{*}{ PSS at 12 months } & $\mathrm{N}-\mathrm{W}$ & 21 & 15.14 & 7.425 & .373 & 32 & .712 \\
\hline & W & 13 & 14.23 & 6.016 & & & \\
\hline \multirow[t]{2}{*}{ STAI at birth } & $\mathrm{N}-\mathrm{W}$ & 24 & 9.29 & 3.724 & -2.367 & 38 & .023 \\
\hline & W & 16 & 12.25 & 4.091 & & & \\
\hline \multirow{2}{*}{ STAI at 40 weeks PMA } & $\mathrm{N}-\mathrm{W}$ & 23 & 8.17 & 2.640 & -1.902 & 36 & .073 \\
\hline & $\mathrm{W}$ & 15 & 11.00 & 5.345 & & & \\
\hline \multirow[t]{2}{*}{ STAI at 6 months } & $\mathrm{N}-\mathrm{W}$ & 19 & 9.16 & 3.804 & -.281 & 30 & .781 \\
\hline & W & 13 & 9.54 & 3.711 & & & \\
\hline \multirow[t]{2}{*}{ STAI at 12 months } & $\mathrm{N}-\mathrm{W}$ & 21 & 9.43 & 3.586 & -.534 & 32 & .597 \\
\hline & $\mathrm{W}$ & 13 & 10.08 & 3.174 & & & \\
\hline \multirow[t]{2}{*}{ SFPCS at 40 weeks PMA } & $\mathrm{N}-\mathrm{W}$ & 23 & 46.94 & 7.496 & .368 & 36 & .715 \\
\hline & $\mathrm{W}$ & 15 & 46.08 & 6.343 & & & \\
\hline \multirow[t]{2}{*}{ Physical Exercise at 40 weeks PMA } & N-W & 23 & 16.17 & 13.300 & -.452 & 36 & .654 \\
\hline & $\mathrm{W}$ & 15 & 18.20 & 13.801 & & & \\
\hline \multirow{2}{*}{ SFMCS at 40 weeks PMA } & $\mathrm{N}-\mathrm{W}$ & 23 & 49.44 & 10.443 & .221 & 36 & .827 \\
\hline & $\mathrm{W}$ & 15 & 48.65 & 11.027 & & & \\
\hline \multirow[t]{2}{*}{ SFPCS at 6 months } & $\mathrm{N}-\mathrm{W}$ & 19 & 47.41 & 10.279 & .440 & 30 & .663 \\
\hline & $\mathrm{W}$ & 13 & 45.96 & 7.040 & & & \\
\hline \multirow[t]{2}{*}{ Physical Exercise at 6 months } & $\mathrm{N}-\mathrm{W}$ & 18 & 15.00 & 10.944 & -.272 & 29 & .788 \\
\hline & $\mathrm{W}$ & 13 & 16.15 & 12.615 & & & \\
\hline \multirow[t]{2}{*}{ SFMCS at 6 months } & $\mathrm{N}-\mathrm{W}$ & 19 & 48.19 & 9.450 & -.191 & 30 & .850 \\
\hline & $\mathrm{W}$ & 13 & 48.86 & 10.012 & & & \\
\hline \multirow[t]{2}{*}{ SFPCS at 12 months } & $\mathrm{N}-\mathrm{W}$ & 21 & 48.99 & 8.444 & .459 & 32 & .649 \\
\hline & $\mathrm{W}$ & 13 & 47.67 & 7.564 & & & \\
\hline \multirow[t]{2}{*}{ Physical Exercise at 12 months } & $\mathrm{N}-\mathrm{W}$ & 21 & 10.67 & 10.504 & -.647 & 32 & .522 \\
\hline & $\mathrm{W}$ & 13 & 13.38 & 13.908 & & & \\
\hline \multirow[t]{2}{*}{ SFMCS at 12 months } & $\mathrm{N}-\mathrm{W}$ & 21 & 48.33 & 9.741 & .331 & 32 & .742 \\
\hline & $\mathrm{W}$ & 13 & 47.22 & 9.057 & & & \\
\hline \multirow[t]{2}{*}{ PSI at 6 months } & $\mathrm{N}-\mathrm{W}$ & 19 & 56.74 & 13.556 & -.215 & 30 & .831 \\
\hline & $\mathrm{W}$ & 13 & 58.23 & 25.580 & & & \\
\hline PSI at 12 months & $\mathrm{N}-\mathrm{W}$ & 21 & 65.52 & 20.024 & 1.240 & 32 & .224 \\
\hline & $\mathrm{W}$ & 13 & 57.69 & 13.634 & & & \\
\hline
\end{tabular}

Note. CES-D = the Center for Epidemiologic Studies Depression, PMA = postmenstrual age, PSI = Parenting Stress Index, PSS = Perceived Stress Scale, SFMCS = the 12 Item Short Form Health Survey Mental Composite

\section{Maternal Mental and Physical Health During the First Year After Birth}

As shown in Table 3, the means of the CES$\mathrm{D}$ at the four time points were positively correlated each other. The means of the PSS at the four time points were positively correlated with each other except PSS at birth and PSS at 12 months $(r=.245, p$
$=.16)$. The means of the STAI at the four time points were positively correlated with each other except STAI at birth and STAI at 12 months $(r=.051, p=$ $0.78)$ as well as STAI at 40 weeks PMA and STAI at 6 months $(r=.348, p=.06)$. The means of the maternal mental composite score on the SF-12 (SFMCS) at three time points (40 weeks PMA, 6 months, and 12 months) were positively correlated with each other 
except SFMCS at 40 weeks PMA and SFMCS at 12 months $(r=.291, p=.09)$. The means of the PSI at two time points ( 6 and 12 months) were not correlated each other $(r=.163, p=.38)$.

The means of the maternal physical composite score on the SFPCS at three time points (40 weeks PMA, 6 months, and 12 months) were positively correlated with each other except SFMCS at 40 weeks PMA and SFMCS at 12 months $(r=.336, p=.05)$. The means of physical exercise at the three time points were positively correlated with each other except physical exercise at 40 weeks and physical exercise at 6 months $(r=.262, p=.16)$.

\section{Associations Between Physical Exercise and Maternal Mental and Physical Health}

As shown in Table 4, physical exercise was not associated with maternal mental health (including depressive symptoms, anxiety, mental composite score on the SF-12, and parenting stress), except for PSS at 12 months. Higher physical exercise scores at 12 months were significantly related to lower levels of maternal perceived stress at 12 months. Mothers reported lower stress levels at 12 months when they exercised more at 12 months.

\section{Maternal Mental and Physical Health Between Non-White Mothers and White Mothers}

As shown in Table 5, the means of maternal mental health (depressive symptoms, perceived stress, and parenting stress) did not differ between non-White mothers and White mothers, except that White mothers reported a higher level of anxiety than non-White mothers at birth. The mean scores of the STAI were higher in White mothers than non-White mothers at the four time points. On the other hand, the means of maternal physical health (SFPCS on the SF-12) did not differ between non-White mothers and White mothers at three time points (at 40 weeks PMA, 6 months, and 12 months).

\section{Discussion}

The study supports our hypotheses that maternal steroid hormonal levels, testosterone and cortisol, were predictive of maternal mental and physical health problems during the first year after birth. In addition, we found that physical exercise reduced maternal perceived stress. However, unexpectedly, maternal health deteriorated when their VLBW infants grew up. Also, it was noted that White mothers experienced more anxiety than non-White mothers throughout the study, especially at birth. Physical health appeared to be better in non-White mothers including Asian mothers than White mothers; however, non-White mothers tended to exercise less than White mothers.

Little is known currently as to how maternal cortisol and testosterone are associated with maternal health outcomes during the first year after birth. This is when mothers of VLBW preterm infants usually experience more difficulties than mothers of normal birthweight full-term infants in parenting. We found that testosterone levels were positively associated with maternal health problems and more predictive than cortisol levels. Regardless of maternal race, maternal mental health worsened whereas maternal physical health improved when the infant grew up.

As maternal testosterone and cortisol levels are known to parallel each other (Cho et al., 2012; Sarkar et al., 2007), we expected that both biomarkers would be consistently and negatively associated with maternal health outcomes. However, only high maternal testosterone levels were found to be consistently related to maternal health problems. Mothers with high testosterone levels reported greater depressive symptoms, more perceived stress, more parenting stress, and worse mental and physical health status, which was indicated by lower scores on the mental and physical composite scores on the SF-12.

Unexpectedly, high maternal cortisol levels were not consistently associated in a negative manner with maternal mental and physical health problems. Mothers with high cortisol levels reported greater anxiety at 40 weeks PMA and 6 months, but the mothers reported better mental and physical health status as indicated by higher scores on the mental and physical composite scores on the SF-12. Mothers with high cortisol levels also reported that they exercised more regularly than mothers with low cortisol levels. These findings showed that testosterone may be more predictive of maternal mental and physical health than cortisol.

As cortisol is considered a stress hormone, this biomarker has been commonly used to assess mental health status. However, the associations between women's cortisol levels and their mental health status have been inconsistent over time between nonWhite and White women. For example, non-White women are known to have greater depressive symptoms and stress than White women but the cortisol levels of non-White women early in the morning are shown to be lower than those of White women, and diurnal cortisol rhythms are flatter in non-White women than in White women (DeSantis, Adam, Hawkley, Kudielka, \& Cacioppo, 2015). This finding is similar to another study with non-White women that found that non-depressed non-obese women showed significantly higher cortisol levels than women with 
depression and obesity (Luiza, Gallaher, \& Powers, 2015). These results from both studies appear to support that testosterone may be more predictive of maternal mental health than cortisol.

In the current study, high maternal cortisol levels at 6 months were more predictive of better maternal mental and physical health at 6 and 12 months. Thus, there might be a transition point between 40 weeks PMA and 6 months for cortisol to become a positive factor for maternal health. At present, we are not able to offer any possible explanation for this possibility.

Although maternal mental health worsened during the first year after birth, maternal physical health improved as the VLBW infants grew. Thus mothers of VLBW infants are indeed at high-risk for maintaining optimal mental health possibly because of the difficulties and the extra burden of parenting than mothers of normal birth weight full-term infants. Mothers of VLBW infants appeared to experience fewer mental health problems between 40 weeks PMA and 6 months than at birth regardless of maternal race and infant gender. However, the mothers reported more mental health problems including perceived stress, anxiety, and parenting stress between 6 to 12 months. These findings show that mothers of VLBW infants need help from health care providers and family members to maintain or improve their mental health during the first year after birth, especially 6 months after.

Mothers showed improvement in physical health from 40 weeks PMA to 12 months although the physical health status was less favorable than that of the U.S. population and the participants in the Utah Health Status Survey. These findings show that mothers of VLBW infants might be physically overwhelmed throughout the first year, especially immediately after birth.

We found that physical exercise reduced maternal perceived stress at 12 months. The mothers exercised less as the infant became older, possibly because of a lack of time. Thus, mothers need to be encouraged to do physical exercise regularly to reduce their stress. We were not able to find the effects of physical exercise on other mental health problems including depressive symptoms, anxiety, and parenting stress. If maternal perceived stress (e.g., how often have you felt nervous and stressed) differs from parenting stress (e.g., having a child caused problems with spouse), future studies need to investigate possible reasons for increasing maternal perceived stress.

Although mothers exercised less and less from birth to 12 months, our data show that physical exercise was the only factor that could improve maternal mental health, especially reduce perceived stress. Many studies have shown significant benefits of physical exercise for women with mental health problems (Heesch, van Gellecum, Burton, van Uffelen, \& Brown, 2015; Howard \& Khalifeh, 2015; Pavey, Burton, \& Brown, 2015). Mothers of VLBW infants appeared to have less time for physical exercise when their infants were older but these mothers need to be strongly encouraged to exercise to improve their physical as well as their mental health. Structured group programs are effective in improving mental health problems; however, accumulation of moderateintensity activity such as walking would be helpful and would be most appropriate for these mothers. Walking programs through lifestyle modification also have shown great benefits for improving mental health (Richardson et al., 2005).

White mothers were more likely to experience depressive symptoms than non-White mothers whereas non-White mothers were more likely to experience perceived stress than White mothers throughout the study. However, note that these differences on the CES-D scores and the PSS scores were not statistically significant. White mothers also reported more anxiety than non-White mothers throughout the four time points, especially at birth. White mothers might feel upset and worried more about having a VLBW preterm infant than non-White mothers, especially immediately after birth. The mean scores of the SFPCS, physical health, were greater in non-White mothers compared to White mothers at the three time points. However, the mean scores of physical exercise were greater in White mothers than non-White mothers at the three time points. If a lack of physical exercise in non-White mothers is related to culture rather than physical strength, non-White mothers including Asian American mothers need to be encouraged to exercise on a daily basis.

\section{Limitations}

This study contains several limitations. Data collection at the four time points might increase the power but the sample size $(N=40)$ might not be enough to support our hypotheses. Thus the findings are potentially subject to lack of power or Type II error. Most mothers $(95 \%)$ received prenatal glucocorticoid treatment before birth and enrollment that was not avoidable. However, the treatment might affect maternal testosterone and cortisol levels. We used self-report to assess maternal mental and physical health that might over or underestimate the problems. It would be interesting to compare the study results between mothers of normal birth weight full-term infants and mothers of VLBW preterm infants. 


\section{Conclusion}

The assessment of maternal health problems using hormonal biomarkers along with self-report was more informative than using self-report alone. The levels of testosterone and cortisol have been found to be positively correlated in women (Cho et al., 2012; Sarkar et al., 2007). Thus, we had expected both testosterone and cortisol levels might be equally associated with maternal health outcomes. However, testosterone was more predictive of maternal mental as well as physical health than cortisol. When VLBW infants grew up during the first year after birth, mothers improved their physical health but showed deteriorated mental health. As physical exercise was found to be helpful in improving mental health, especially to reduce perceived stress, mothers of VLBW infants should be encouraged to exercise regularly regardless of race.

\section{Declaration of Conflicting Interests}

The authors declare no conflicts of interest.

\section{Funding}

The preparation of this manuscript was supported by the National Institute of Child Health and Human Development (R01HD076871), National Institutes of Health to the first author.

\section{References}

Altindag, O., Altindag, A., Asoglu, M., Gunes, M., Soran, N., \& Deveci, Z. (2007). Relation of cortisol levels and bone mineral density among premenopausal women with major depression. International Journal of Clinical Practice, 61, 416-420. doi:10.1111/j.1742-1241.2006.01276.x

Andreou, E., Alexopoulos, E. C., Lionis, C., Varvogli, L., Gnardellis, C., Chrousos, G. P., \& Darviri, C. (2011). Perceived Stress Scale: Reliability and validity study in Greece. International Journal of Environmental Research and Public Health, 8, 3287-3298. doi:10.3390/ijerph8083287

Bauman, A. E. (2004). Updating the evidence that physical activity is good for health: An epidemiological review 2000-2003. Journal of Science and Medicine in Sport, 7(1 Suppl), 6-19. doi:10.1016/S1440-2440(04)80273-1

Bolten, M. I., Wurmser, H., Buske-Kirschbaum, A., Papousek, M., Pirke, K. M., \& Hellhammer, D. (2011). Cortisol levels in pregnancy as a psychobiological predictor for birth weight. Archives of Womens' Mental Health, 14, 33-41. doi:10.1007/s00737-010-0183-1

Brockington, I., Butterworth, R., \& Glangeaud-Freudenthal, N. (2016). An international position paper on mother-infant (perinatal) mental health, with guidelines for clinical practice. Archives of Women's Mental Health. doi:10.1007/s00737-0160684-7

Brown, W. J., Burton, N. W., \& Rowan, P. J. (2007). Updating the evidence on physical activity and health in women. American Journal of Preventive Medicine, 33, 404-411. doi:10.1016/j.amepre.2007.07.029

Carlsen, S. M., Jacobsen, G., \& Romundstad, P. (2006). Maternal testosterone levels during pregnancy are associated with offspring size at birth. European Journal of Endocrinology, 155, 365-370. doi:10.1530/eje.1.02200

Chen, T., Surcel, H. M., Lundin, E., Kaasila, M., Lakso, H. A., Schock, H., . . Lukanova, A. (2011). Circulating sex steroids during pregnancy and maternal risk of non-epithelial ovarian cancer. Cancer Epidemiology, Biomarkers and Prevention, 20, 324336. doi:10.1158/1055-9965.EPI-10-0857

Cho, J., Holditch-Davis, D., \& Miles, M. S. (2008). Effects of maternal depressive symptoms and infant gender on the interactions between mothers and their medically at-risk infants. Journal of Obstetric, Gynecologic, and Neonatal Nursing, 37, 58-70. doi:10.1111/j.1552-6909.2007.00206.x

Cho, J., Carlo, W. A., Su, X., \& McCormick, K. L. (2012). Associations between salivary testosterone and cortisol levels and neonatal health and growth outcomes. Early Human Development, 88, 789795. doi:10.1016/j.earlhumdev.2012.05.002

Cutolo, M. (2009). Androgens in rheumatoid arthritis: When are they effectors? Arthritis Research \& Therapy, 11, 126. doi:10.1186/ar2804

DeSantis, A. S., Adam, E. K., Hawkley, L. C., Kudielka, B. M., \& Cacioppo, J. T. (2015). Racial and ethnic differences in diurnal cortisol rhythms: Are they consistent over time? Psychosomatic Medicine, 77, 6-15. doi:10.1097/PSY.0000000000000131

Feise, R. J. (2002). Do multiple outcome measures require p-value adjustment? BMC Medical Research Methodology, 2, 8.

Granger, D. A., Shirtcliff, E. A., Zahn-Waxler, C., Usher, B., Klimes-Dougan, B., \& Hastings, P. (2003). Salivary testosterone diurnal variation and psychopathology in adolescent males and females: Individual differences and developmental effects. Development and Psychopathology, 15, 431-449. doi:10.1017/S0954579403000233

Hamer, M., \& Steptoe, A. (2012). Cortisol responses to mental stress and incident hypertension in healthy men and women. Journal of Clinical Endocrinology and Metabolism, 97, E29-34. doi: 10.1210/jc.2011-2132

Haskett, M. E., Ahern, L. S., Ward, C. S., \& Allaire, J. C. (2006). Factor structure and validity of the parenting stress index-short form. Journal of Clinical Child and Adolescent Psychology, 35, 302312. doi:10.1207/s15374424jccp3502_14

Heesch, K. C., van Gellecum, Y. R., Burton, N. W., van Uffelen, J. G. Z., \& Brown, W. J. (2015). Physical activity, walking, and quality of life in women with depressive symptoms. American Journal of Preventive Medicine, 48, 281-291. doi:10.1016/j.amepre.2014.09.030 
Holditch-Davis, D., Schwartz, T., Black, B., \& Scher, M. (2007). Correlates of mother-premature infant interactions. Research in Nursing \& Health, 30, 333-346. doi:10.1002/nur.20190

Howard, L. M., \& Khalifeh, H. (2015). Randomised controlled trial of facilitated exercise plus usual care versus usual care only as a treatment for women with a depressive disorder in the first six postnatal months. Evidence-Based Mental Health, 18, 123. doi:10.1136/eb-2015-102140

Howe, T. H., Sheu, C. F., Wang, T. N., \& Hsu, Y. W. (2014). Parenting stress in families with very low birth weight preterm infants in early infancy. $R e$ search in Developmental Disabilities, 35, 17481756. doi:10.1016/j.ridd.2014.02.015

Janisse, H. C., Nedd, D., Escamilla, S., \& Nies, M. A. (2004). Physical activity, social support, and family structure as determinants of mood among European-American and African-American women. Women \& Health, 39, 101-116. doi:10.1300/J013v39n01_06

Kim, S., Popkin, B. M., Siega-Riz, A. M., Haines, P. S., \& Arab, L. (2004). A cross-national comparison of lifestyle between China and the United States, using a comprehensive cross-national measurement tool of the healthfulness of lifestyles: The Lifestyle Index. Preventive Medicine, 38, 160-171. doi:10.1016/j.ypmed.2003.09.028

King, J. T., Jr., Horowitz, M. B., Kassam, A. B., Yonas, H., \& Roberts, M. S. (2005). The short form-12 and the measurement of health status in patients with cerebral aneurysms: Performance, validity, and reliability. Journal of Neurosurgery, 102, 489494. doi:10.3171/jns.2005.102.3.0489

Lesage, F. X., Berjot, S., \& Deschamps, F. (2012). Psychometric properties of the French versions of the Perceived Stress Scale. International Journal of Occupational Medicine and Environmental Health, 25, 178-184. doi:10.2478/s13382-0120024-8

Luiza, J. W., Gallaher, M. J., \& Powers, R. W. (2015). Urinary cortisol and depression in early pregnancy: Role of adiposity and race. BMC Pregnancy and Childbirth, 15, 30. doi:10.1186/s12884-0150466-7

Manikkam, M., Crespi, E. J., Doop, D. D., Herkimer, C., Lee, J. S., Yu, S., . . Padmanabhan, V. (2004). Fetal programming: Prenatal testosterone excess leads to fetal growth retardation and postnatal catch-up growth in sheep. Endocrinology, 145, 790-798. doi:10.1210/en.2003-0478

Marteau, T. M., \& Bekker, H. (1992). The development of a six-item short-form of the state scale of the Spielberger State-Trait Anxiety Inventory (STAI). British Journal of Clinical Psychology, 31, 301-306. doi:10.1111/j.20448260.1992.tb00997.x

McGregor, B. A., \& Antoni, M. H. (2009). Psychological intervention and health outcomes among women treated for breast cancer: A review of stress pathways and biological mediators. Brain, Behavior, and Immunity, 23, 159-166. doi:S0889-

1591(08)00318-8 [pii]10.1016/j.bbi.2008.08.002
Mezzullo, M., Fanelli, F., Fazzini, A., Gambineri, A., Vicennati, V., Di Dalmazi, G., . . . Pasquali, R. (2016). Validation of an LC-MS/MS salivary assay for glucocorticoid status assessment: Evaluation of the diurnal fluctuation of cortisol and cortisone and of their association within and between serum and saliva. The Journal of Steroid Biochemistry and Molecular Biology, 163, 103 112. doi:10.1016/j.jsbmb.2016.04.012

Office of Public Health Assessment (2002). Overview of the 2001 Health Status Survey: 2001 Utah Health Status Survey Report. Salt Lake City, UT: Utah Department of Health. Retrieved from http://health.utah.gov/ibis-ph/opha_pubs.html

Owen, D. J., Wood, L., Tomenson, B., Creed, F., \& Neilson, J. P. (2016). Social stress predicts preterm birth in twin pregnancies. Journal of Psychosomatic Obstetrics and Gynaecology, 11, 110. Advance online publication. doi:10.1080/0167482X.2016.1235146

Papacosta, E., Gleeson, M., \& Nassis, G. P. (2013). Salivary hormones, IgA, and performance during intense training and tapering in judo athletes. Journal of Strength and Conditioning Research, 27, 2569-2580. doi:10.1519/JSC.0b013e31827fd85c

Pavey, T. G., Burton, N. W., \& Brown, W. J. (2015). Prospective relationships between physical activity and optimism in young and mid-aged women. Journal of Physical Activity \& Health, 12, 915923. doi:10.1123/jpah.2014-0070

Penedo, F. J., \& Dahn, J. R. (2005). Exercise and well-being: A review of mental and physical health benefits associated with physical activity. Current Opinion in Psychiatry, 18, 189-193. doi:10.1097/00001504-200503000-00013

Radloff, L. S. (1977). The CES-D scale: A self-report depression scale for research in the general population. Applied Psychological Measurement, 1, 385-401. doi:10.1177/014662167700100306

Redshaw, M., \& Henderson, J. (2016). Who is actually asked about their mental health in pregnancy and the postnatal period? Findings from a national survey. BMC Psychiatry, 16, 322. doi:10.1186/s12888-016-1029-9

Richardson, C. R., Faulkner, G., McDevitt, J., Skrinar, G. S., Hutchinson, D. S., \& Piette, J. D. (2005). Integrating physical activity into mental health services for persons with serious mental illness. Psychiatric Services, 56, 324-331. doi:10.1176/appi.ps.56.3.324

Rosner, W., Auchus, R. J., Azziz, R., Sluss, P. M., \& Raff, H. (2007). Position statement: Utility, limitations, and pitfalls in measuring testosterone: An Endocrine Society position statement. Journal of Clinical Endocrinology and Metabolism, 92, 405-413. doi:10.1210/jc.2006-1864

Sarkar, P., Bergman, K., Fisk, N. M., O'Connor, T. G., \& Glover, V. (2007). Amniotic fluid testosterone: Relationship with cortisol and gestational age. The Clinical Endocrinology, 67, 743-747. doi:10.1111/j.1365-2265.2007.02955.x 
Soriguer, F., Rubio-Martin, E., Fernandez, D., Valdes, S., Garcia-Escobar, E., Martin-Nunez, G. M., .. . Rojo-Martinez, G. (2012). Testosterone, SHBG and risk of type 2 diabetes in the second evaluation of the Pizarra cohort study. European Journal of Clinical Investigation, 42, 79-85. doi:10.1111/j.1365-2362.2011.02559.x

van der Bij, A. K., de Weerd, S., Cikot, R. J., Steegers, E. A., \& Braspenning, J. C. (2003). Validation of the dutch short form of the state scale of the Spielberger State-Trait Anxiety Inventory: Considerations for usage in screening outcomes. Community Genetics, 6, 84-87. doi:10.1159/000073003

Ware, J., Jr., Kosinski, M., \& Keller, S. D. (1996). A 12Item Short-Form Health Survey: Construction of scales and preliminary tests of reliability and validity. Medical Care, 34, 220-233. 